\title{
Tick paralysis
}

\author{
Sylvain A. Lother MD, Laura Haley MD
}

Cite as: CMAJ 2017 October 30;189:E1341. doi: 10.1503/cmaj.170868

A healthy and active 83-year-old man presented to the emergency department after five days of paresthesias, followed by rapidly progressive ataxia, weakness and severely impaired ambulation. Symptoms started eight days after he worked at his cabin in the ThompsonNicola region of British Columbia, Canada.

Upon examination, the patient was afebrile with normal vital signs and mentation. He had diplopia, marked dysarthria, four-limb weakness, dysmetria, areflexia, and reduced vibration sense and proprioception distally. We removed an engorged tick that was adherent to his mid-back (Figure 1A). Routine analyses of blood and cerebrospinal fluid were normal. The BC Centre for Disease Control Public Health Laboratory used sterotactic microscopy to identify it as a female Rocky Mountain wood tick (Dermacentor andersoni; Figure 1B and 1C).

Within two hours of removal of the tick, the patient's reflexes had returned to normal, and his strength had substantially improved. Complete neurologic recovery occurred within 72 hours.

Tick paralysis, a neurotoxidrome, occurs after tick attachment, engorgement and injection of potent tick salivary toxins. Women and children are most affected. Ticks hide in long body hair, skin folds and difficult-to-reach areas. Most cases occur from March to July when ticks are most active. Four to seven days after tick attachment, symptoms begin with malaise and weakness, followed by progressive neurologic deficits. Paralysis ensues in a mean of 1.4 (range 1-10) days. ${ }^{1}$ Suggestive symptoms include diplopia, dysphagia, dysarthria, ataxia and ascending weakness; it is frequently misdiagnosed as Guillain-Barré syndrome. ${ }^{1,2}$ Other diagnostic considerations include botulism, diphtheria and organophosphate poisoning.

Over 40 tick species are capable of paralyzing humans and animals. ${ }^{3}$ Dermacentor andersoni in western Canada and the Pacific northwest, Dermacentor variabilis in the southeastern United States and Ixodes holocyclus in eastern Australia are the most common. ${ }^{1} \mathrm{~A}$ thorough dermatologic examination is necessary in patients with flexic weakness and dysmetria, particularly during tick season. Treatment of tick bites requires prompt removal of the tick with tweezers. The tick should be grabbed close to its mouth and pulled up gently but firmly without jerking or twisting; the skin should be disinfected. ${ }^{4}$

Typically, rapid neurologic recovery ensues, usually within minutes to hours, and full recovery usually occurs after 1.5 (range 1-2.5) days. ${ }^{1}$

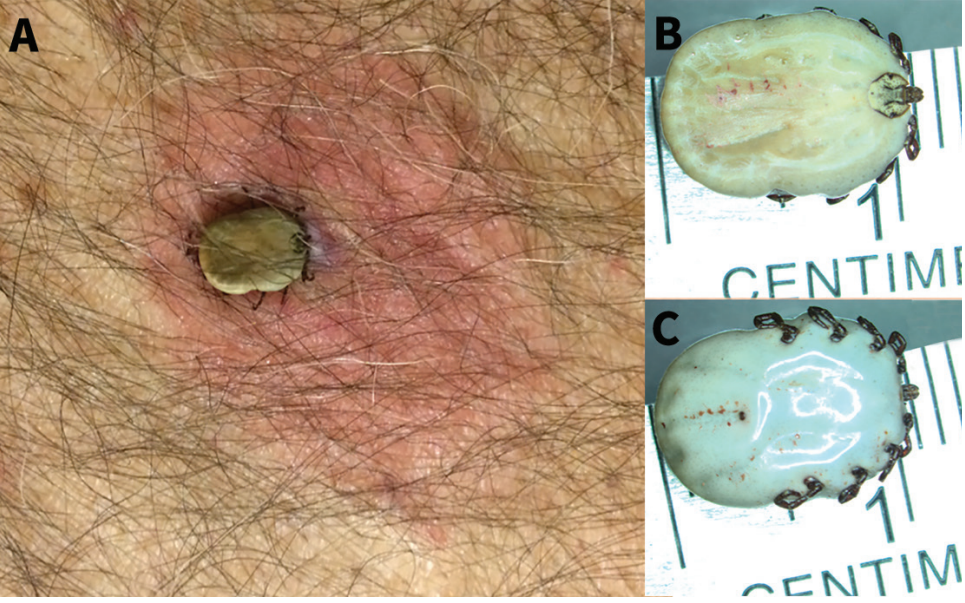

Figure 1: (A) An engorged tick adherent to the mid-back of an 83-year-old man, with $\mathrm{cm}$ of surrounding erythema centred around the site of attachment. Stereotactic microscopy views of the (B) dorsal and (C) ventral aspects showing the characterisic appearance of a female Rocky Mountain wood tick (Dermacentor andersoni) original magnification $\times 12.5$ ).

\section{References}

1. Diaz JH. A 60-year meta-analysis of tick paralysis in the United States: a predictable, preventable, and often misdiagnosed poisoning. J Med Toxicol 2010;6:15-21.

2. Crawford $P$, Mitchell D. Tick paralysis as a cause of autonomic dysfunction in a 57-year-old female. South Med J 2009;102:190-2.

3. Gothe R, Kunze K, Hoogstraal H. The mechanisms of pathogenicity in the tick paralyses. J Med Entomol 1979;16:357-69.

4. Needham GR. Evaluation of five popular tick removal methods for tick removal. Pediatrics 1985;75:997-1002.

\section{Competing interests: None declared.}

This article has been peer reviewed.

The authors have obtained patient consent.

Affiliations: Sections of Infectious Diseases (Lother) and Neurology (Haley), Department of Internal Medicine, The University of British Columbia; Vancouver General Hospital (Lother); Gordon and Leslie Diamond Health Care Centre (Haley), Vancouver, BC

Acknowledgements: The authors thank the BC Centre for Disease Control Public Health Laboratory, Parasitology section, who performed the tick identification and photography, and local tick experts, Dr. William Bowie and Dr. Muhammad Moreshed, who were consulted on this case.

Correspondence to: Sylvain Lother, sa.lother@me.com 